\title{
Balloon Occlusion Tests and Therapeutic Vessel Occlusions Revisited: When, When Not, and How
}

T he concept of therapeutic occlusion of arteries to and in the brain is old, with Cooper describing a carotid artery ligation performed in $1805,{ }^{1}$ and Matas, a vertebral artery sacrifice in $1893 .^{2}$ The potentially deleterious effect of losing a carotid artery was even known in ancient Greece, because they named this artery "karos," meaning to stupefy or plunge into deep sleep. Leonardo da Vinci (1452-1519) described the vessels of the neck and suggested that compression of the carotid artery, which at that time usually was called the "soporal artery," could rapidly lead to unconsciousness. Unselected carotid artery sacrifice without revascularization will lead to potentially lethal cerebral infarction in about two-thirds of individuals. Sacrifice or damage to the vertebral artery was earlier considered a "no man's land" as reflected in the citation by Sanson from 1836: "The vertebral artery cannot be ligated, on account of its great depth, nor compressed, because of the osseous canal which protects it; it can still less be cauterized. The wounds of this vessel are beyond the resources of art" (cited in $\left.\operatorname{Matas}^{2}\right)$.

The apprehension of the risk of therapeutic artery sacrifice has urged preprocedural risk evaluations, which, since Serbinenko's invention of balloon catheters in the early $70 \mathrm{~s},{ }^{3}$ usually are performed by angiographic balloon test occlusion (BTO). BTO with clinical surveillance considerably reduced the incidence of postoperative stroke compared with unselected carotid artery sacrifice but still carries a $5 \%-40 \%$ stroke rate. ${ }^{4,5}$ In an attempt to enhance the prognostic validity of a BTO with clinical surveillance, a large battery of adjuvant assessments evolved. These include hypotensive challenges, stump pressure measurements, electroencephalography and evoked potentials monitoring, cerebral oximetry by using near-infrared spectroscopy, blood velocity measurements applying transcranial Doppler ultrasonography (TCD), evaluation of cerebral blood flow with technetium Tc99m hexamethylpropyleneamine oxime single-photon emission CT, positron-emission tomography, xenon-enhanced CT, and/or MR perfusion imaging.

This vast diversity of BTO protocols reflects the lack of a consensus as to a specified method clearly superior to others. This may be due to the persisting significant number of failures to predict hemodynamic stroke at carotid artery sacrifice, despite advanced but not necessarily adequate protocols. For instance, relative changes in $\mathrm{CBF}$ comparing the occluded with the nonoccluded hemisphere (qualitative evaluation) may introduce an error due to the nonoccluded side undergoing changes in CBF during the BTO as well (ie, the contralateral hemisphere cannot be used as a constant reference). Hence, up to $20 \%$ of patients may not tolerate ICA sacrifice, even though having symmetric BTO scan findings. ${ }^{6-8}$ Quantitative CBF analysis during BTO using xenon-enhanced CT lowers the rate of false-negative BTO findings but they still remain between 3\% and 10\%. ${ }^{5,9,10}$

In addition to the imperfection of the predictive value of many BTO protocols, there is a procedural risk of 3\%-4\% in performing a BTO, ${ }^{11-13}$ which needs to be addressed. Many complications are related to intimal damage, potentially resulting in arterial dissection and/or pseudoaneurysm formation and consecutive thromboembolism. ${ }^{12}$ A common factor for most carotid artery BTO protocols is occlusion times ranging from 15 to 30 minutes along with the need for transporting the patient, with catheters in place, to a different suite. The rate of complications increases with the length of the angiographic procedure $^{12}$ or, in other words, the longer the BTO occlusion time, the higher the procedural risk.

In light of this reality, there is actually no good reason to still perform prolonged carotid artery occlusion tests because there are very swift BTO methods requiring no more than 60-90 seconds of occlusion time: The venous phase BTO, ${ }^{14,15}$ with or without ultraswift TCD evaluation, ${ }^{16}$ hence carries a very low procedural risk $(0 \%-0.7 \%)$ (ie, it does not exceed the risk of diagnostic cerebral angiography per se). In addition, the positive predictive value of the latter method of BTO, ranging from $98 \%$ to $100 \%$, is unsurpassed by any other protocol. Being nonclinical, this type of BTO does not lose any of its high predictive value when performed with the patient under general anesthesia. Venous phase BTO requires bilateral catheterization, but an increase in inguinal complications has yet not been reported. This method measures the synchronicity (not symmetry!) of venous filling between the occluded and nonoccluded hemispheres. A delay of $<0.5$ seconds in venous filling is considered safe for permanent vessel occlusion. ${ }^{14}$ Carotid 
artery sacrifice can actually be performed uneventfully in patients with a delay in venous filling of $<2$ seconds, whereas patients with a delay of 3 seconds may be prone to hemodynamic ischemia in conjunction with hypotensive episodes. ${ }^{15}$

The venous phase BTO can be further enhanced by concomitant TCD evaluation, ${ }^{16}$ which can depict a subgroup of individuals who fail the venous phase test but still can safely undergo carotid artery sacrifice when passing the Doppler criteria. ${ }^{16}$ Given the unexcelled level of safety and predictive value of TCD-guided venous phase BTO, this procedure should be considered "state of the art" in the preoperative evaluation of therapeutic carotid artery occlusion. It is good practice to perform a baseline TCD before the BTO, including a digital carotid compression test. If the ipsilateral middle cerebral artery velocity drops to $<30 \%$ of baseline on carotid compression, an angiographic BTO can be omitted because it is obvious that the cerebral collateral capacity is insufficient. This does, though, not apply if the underlying pathology is a carotico-cavernous fistula or a highly vascularized neck or skull base tumor.

Regarding BTO in the vertebrobasilar circulation, there is not yet a reliable ultraswift protocol. This test is still clinical, in the 15to 30-minute range. BTO in the vertebrobasilar circulation should only be performed with bilateral vertebral artery (VA) occlusion, unless the patient has merely 1 sole vertebral artery (ie, the contralateral vertebral artery is aplastic, occluded, or supplies its posterior inferior cerebellar artery [PICA] only). In other words, vertebral BTO should only be performed to test the collateral capacity of the circle of Willis, not to visualize retrograde ipsilateral vertebral filling alone. With 2 vertebral arteries joining at the vertebrobasilar junction, a unilateral vertebral BTO is obsolete, even if dominant vertebral artery sacrifice is planned. The article of Zoarski and Seth ${ }^{17}$ in the present issue of the American Journal of Neuroradiology provides further evidence of the feasibility of permanent occlusion, even of a dominant vertebral artery, without a preceding BTO.

Bilateral VA BTO can be well-tolerated, even in the presence of 2 very slim posterior communicating arteries (PcomAs) or a single PcomA only (ie, the anatomy of the circle of Willis cannot always predict the feasibility of therapeutic bilateral vertebral [or basilar] occlusion). With TCD, insonation of the P1 segment will show an increase and reversal of flow velocity with a functional PcomA on bilateral VA BTO, whereas there is a drop in P2 velocity. The exact limit for this drop with respect to tolerance to bilateral VA or basilar artery occlusion is yet to be established. Nevertheless, concomitant insonation of the P1 or P2 segment during VA BTO is useful because it immediately detects possible slackening of the balloon. The risk of a false-negative test due to incomplete balloon occlusion can hence be reduced, and continuous Doppler surveillance would be superior to repeated contrast injections.

The most common indication for bilateral VA BTO is to test whether flow reversal for a large basilar tip aneurysm that cannot be treated otherwise at reasonable risk is possible. In that situation, the BTO may be performed at a different site (both VAs) than the one on which the actual permanent occlusion will be performed (either VAs pre-/post-PICA or clip ligation across the basilar artery). In general, a BTO should always be performed as close as possible to the point of intended permanent vessel closure. This is, in particular, important in the following situations:

1) Where the ophthalmic artery retrogradely fills the internal carotid artery and supraclinoid ICA occlusion is planned. BTO should then be performed beyond the ophthalmic artery. ${ }^{18}$

2) In carotico-cavernous fistulas that require balloon placement distal to the site of shunting. Ballon placement proximal to the fisula measures the compound effect of the capacity in the circle of Willis and the fistulous shunting.

3) In richly vascularized tumors of the neck or skull-base, the balloon must be placed distal to the tumor in order to prevent steal phenomena. ${ }^{19}$ On common carotid artery occlusion, blood may flow in a retrograde fashion from the ICA to the bifurcation and supply the tumor through the external carotid artery. This will affect the BTO result significantly.

When it comes to therapeutic vessel occlusion, it should be considered as the integrated art of the optimal choice of the site of occlusion; mode of occlusion; risk assessment regarding hemodynamic and thromboembolic events; as precisely as possible, the estimation of success rate compared with other treatment modalities; and finally, postoperative surveillance and care. Such a procedure hence requires interdisciplinary cooperation among endovascular therapists, neurosurgeons, and neurointensive care staff, including those with expertise in cerebral hemodynamics.

The site of occlusion should be chosen from the perspective of maximal therapeutic effect and minimalization of a "blind stump," where thrombus may form in the artery and give raise to thromboembolism. The BTO can provide safety against hemodynamic events but cannot prevent or predict ischemia due to thromboembolism. This is, in particular, true regarding small perforating vessels. Therapeutic vessel occlusion requires antithrombotic prophylaxis with platelet inhibitors (75-mg acetylsalicylic acid) in conjunction with low-molecular heparin. Often, endovascular occlusion techniques may be chosen because they are less invasive than a neurosurgical approach. On the other hand, the latter provides a more precise and "cleaner" occlusion, which can be especially beneficial in therapeutic occlusion in the posterior circulation or whenever secondary thrombosis of perforating arteries is feared. Combined approaches may be chosen, like balloon or coil embolization of one VA proximal to the PICA and clip ligation of the other VA distal to the PICA for flow-reversal treatment of midbasilar or basilar junction aneurysms. Thus, inflow into the aneurysm may be maximally reduced, whereas the "blind stump" is kept at a minimum and the anterior circulation has a smaller additional vascular territory to supply.

The prevailing indications for therapeutic vessel occlusion are giant, fusiform dissecting, or very small (blister) aneurysms. The mode of action is either parent artery occlusion leading to immediate aneurysm flow stagnation and secondary thrombosis with consecutive shrinkage or flow reversal in the parent artery leading to changes in inflow into the aneurysm. In the latter, intra-aneurysmal flow is often preserved on flow reversal and intra-aneurysmal thrombosis may occur in a delayed fashion. An initial increase in intra-aneurysmal flow has been observed but can still result in a favorable long-term out- 
come. Flow reversal in the parent artery is often chosen for giant untreated or failed basilar tip aneurysms. Thrombosis of giant aneurysms may lead to an initial increase in mass effect, which can be alleviated with steroids. Dissecting aneurysms at the PICA branching may respond well to flow reversal on proximal VA occlusion but may also require trap ligation, especially if ruptured. Likewise, the treatment of ruptured blister aneurysms represents a management conundrum. Preferably, these aneurysms should be repaired (surgically or endovascularly) or wrapped in the acute phase. Therapeutic occlusions performed in the acute phase of aneurysm rupture have the potential of vast hemodynamic infarction if complicated by vasospasm (even if the BTO was passed and the collateral circulation was deemed excellent). ${ }^{20}$

Postoperative treatment includes triple or double hypertensive, hypervolemia, hemodilution therapy. The level of hypertensive treatment is individualized in accordance with arterial blood pressures registered during the BTO. Surveillance of the hemodynamic status of the patient is performed with TCD, and both the triple $\mathrm{H}$ therapy and grade of postoperative mobilization can be tailor-made with the aid of Doppler sonography. ${ }^{16}$ This approach allows therapeutic vessel occlusion even in patients with a BTO indicating a border zone for tolerance.

In general, whenever possible, a vessel-preservation approach is preferable over deconstructive solutions. Recently, low-porosity tubular stent-like implants, so-called flow diverters, have evolved. That type of stent may provide complete thrombosis of giant aneurysms without the need to fill the aneurysm with coils (and increase the mass effect) with preservation of the parent vessel. Flow diverters hence may represent another good treatment option for complex aneurysms that usually would be selected for treatment with therapeutic vessel occlusion. However, serious complications in the use of flow diverters have been reported, including stent and perforator occlusions, ipsilateral parenchymal hemorrhage $(8.5 \%),{ }^{21}$ and delayed aneurysm rupture. $^{22,23}$

The combined mortality/morbidity rate connected to the use of flow diverters is approximately $10 \%$ in unruptured aneurysms, ${ }^{22,23}$ which represents a far higher risk than with therapeutic vessel occlusion. Delayed aneurysm rupture does not occur in therapeutic vessel occlusion. The efficacy of flow diverters in terms of total aneurysm occlusion is $73.6 \%{ }^{24}$ and hence lower than that of therapeutic vessel occlusion. The latter is extremely effective, both in terms of short- and long-term results. The fear of de novo aneurysm formation after therapeutic artery occlusion has sometimes been overemphasized. ${ }^{16,22}$ Increased collateral flow and, hence, hemodynamic stress are present only in the acute phase after therapeutic vessel occlusion because the vessel diameter increases due to higher flow, thereby normalizing hemodynamic stress. Cerebral perfusion is not impaired during a prolonged time after ICA sacrifice in patients who passed the BTO. ${ }^{25}$

The evolution of new surgical and endovascular devices and skills in the future may change our treatment armamentarium and preferences, but at present, therapeutic vessel occlusion still is an efficient and safe treatment option, in particular when applying ultraswift venous phase TCD-guided BTO for preprocedural evaluation.

\section{REFERENCES}

1. Cooper A. A case of aneurysm on the carotid artery. Med Chir Trans 1809;1:1-10

2. Matas R. Traumatisms and traumatic aneurysms of the vertebral artery and their surgical treatment with the report of a cured case. Ann Surg 1893;18:477-521

3. Serbinenko FA. Balloon catheterization and occlusion of major cerebral vessels. J Neurosurg 1974;41:125-45

4. Gonzalez CF, Moret J. Balloon occlusion of the carotid artery prior to surgery for neck tumors. AJNR Am J Neuroradiol 1990;11:649-52

5. Linskey ME, Jungreis CA, Yonas H, et al. Stroke risk after abrupt internal carotid artery sacrifice: accuracy of preoperative assessment with balloon test occlusion and stable xenon-enhanced CT. AJNR Am J Neuroradiol 1994;15:829-34

6. Larson JJ, Tew JM Jr, Tomsick TA, et al. Treatment of aneurysms of the internal carotid artery by intravascular balloon occlusion: longterm follow-up of 58 patients. Neurosurgery 1995;36:23-30

7. Lorberboym M, Pandit N, Machac J, et al. Brain perfusion imaging during preoperative temporary balloon occlusion of the internal carotid artery. J Nucl Med 1996;37:415-19

8. Peterman SB, Taylor A Jr, Hoffman JC Jr. Improved detection of cerebral hypoperfusion with internal carotid balloon test occlusion and 99m Tc-HMPAO cerebral perfusion SPECT imaging. AJNR Am J Neuroradiol 1991;12:1035-41

9. de Vries EJ, Sekhar LN, Horton JA, et al. A new method to predict safe resection of the internal carotid artery. Laryngoscope 1990;100:85-88

10. Witt JP, Yonas H, Jungreis C. Cerebral blood flow response pattern during balloon test occlusion of the internal carotid artery. AJNR Am J Neuroradiol 1994;15:847-56

11. Tarr RW, Jungreis CA, Horton JA, et al. Complications of preoperative balloon test occlusion of the internal carotid arteries: experience in 300 cases. Skull Base Surg 1991;1:240-44

12. Mathis JM, Barr JD, Jungreis CA, et al. Temporary balloon test occlusion of the internal carotid artery: experience in 500 cases. AJNR Am J Neuroradiol 1995;16:749-54

13. Zhu W, Tian YL, Zhou LF, et al. Treatment strategies for complex internal carotid artery (ICA) aneurysms: direct ICA sacrifice or combined with extracranial-to-intracranial bypass. World Neurosurg 2011;75:476-84

14. van Rooij WJ, Sluzewski M, Slob MJ, et al. Predictive value of angiographic testing for tolerance to therapeutic occlusion of the carotid artery. AJNR Am J Neuroradiol 2005;26:175-78

15. Abud DG, Spelle L, Piotin M, et al. Venous phase timing during balloon test occlusion as a criterion for permanent internal carotid artery sacrifice. AJNR Am J Neuroradiol 2005;26:2602-09

16. Sorteberg A, Bakke SJ, Boysen M, et al. Angiographic balloon test occlusion and therapeutic sacrifice of major arteries to the brain. Neurosurgery 2008;63:651-60, discussion 660-61

17. Zoarski GH, Seth R. Safety of unilateral endovascular occlusion of the cervical segment of the vertebral artery without antecedent balloon test occlusion. AJNR Am J Neuroradiol 2014;35

18. Lesley WS, Rangaswamy R. Balloon test occlusion and endosurgical parent artery sacrifice for the evaluation and management of complex intracranial aneurysmal disease. J NeuroInterv Surg 2009;1:112-20

19. Sorteberg A, Sorteberg W, Bakke SJ, et al. Varying impact of common carotid artery digital compression and internal carotid artery balloon test occlusion on cerebral hemodynamics. Head Neck 1998;20:687-94

20. Meling TR, Sorteberg A, Bakke SJ, et al. Blood blister-like aneurysms of the internal carotid artery trunk causing subarachnoid 
hemorrhage: treatment and outcome. J Neurosurg 2008; 108:662-71

21. Cruz JP, Chow M, O'Kelly C, et al. Delayed ipsilateral parenchymal hemorrhage following flow diversion for the treatment of anterior circulation aneurysms. AJNR Am J Neuroradiol 2012;33: 603-08

22. van Rooij WJ, Sluzewski M, van der Laak C. Flow diverters for unruptured internal carotid artery aneurysms: dangerous and not yet an alternative for conventional endovascular techniques. AJNR Am J Neuroradiol 2013;34:3-4

23. Kulcsár Z, Szikora I. The ESMINT Retrospective Analysis of Delayed Aneurysm Ruptures after flow diversion (RADAR) study. EJMINT Original Article, 2012: 1244000088. http://www.ejmint. org/original-article/1244000088. Accessed November 4, 2013
24. Becske T, Kallmes DF, Saatsci I, et al. Pipeline for uncoilable or failed aneurysms: results from a multicenter trial. Radiology 2013; 267:858-68

25. Gevers S, Heijtel D, Ferns SP, et al. Cerebral perfusion long term after therapeutic occlusion of the internal carotid artery in patients who tolerated angiographic balloon test occlusion. AJNR Am J Neuroradiol 2012;33:329-35

A. Sorteberg

Department of Neurosurgery Oslo University Hospital The National Hospital-Rikshospitalet Oslo, Norway

http://dx.doi.org/10.3174/ajnr.A3852 